\title{
Value of positive technetium-99m leucocyte scans in predicting intestinal inflammation
}

\author{
P Gibson, M Lichtenstein, N Salehi, G Hebbard, J Andrews
}

\begin{abstract}
The value of positive scans of autologous ${ }^{99 m} \mathrm{Tc}$-labelled leucocytes in the detection of intestinal inflammation was assessed in 29 patients with known inflammatory bowel disease and a high probability of intestinal inflammation and 58 with a low probability of intestinal inflammation comprising 37 with intestinal symptoms and 21 with suspected abscess. Autologous leucocytes were labelled with ${ }^{99 m}$ Tc using either of the established methods. In patients with inflammatory bowel disease, $3 / 13$ with ileocaecal and 1/11 with colonic accumulation of ${ }^{99 m} \mathrm{Tc}$ were incorrectly diagnosed in that there was no histological evidence of inflammation in those regions. In the low probability patients, all 26 positive images were false positives: there were 19 in the ileocaecal and 7 in colonic regions and no differences between the two subgroups. False positives were more common in low probability than high probability patients $(p=0.011)$. The results were independent of the ${ }^{99 m} \mathbf{T c}$ cell labelling technique used. Three false positive patients also had normal scans for ${ }^{11}$ Inlabelled granulocytes. The time of first detection of ${ }^{99 m} \mathbf{T c}$ in the bowel was earlier in true than in false positive patients $(92 \%$ v $33 \%$ within $1 \mathrm{~h}, \mathrm{p}=0.008$ ), the false positive rates in the first hour of imaging being $5 \%$ in high and $15 \%$ in low probability patients. In ${ }^{9 m}$ Tc scintigraphy, the appearance of the isotope in the intestine occurs commonly in the absence of inflammation irrespective of the cell labelling technique used. In high probability patients, imaging at $1 \mathrm{~h}$ can more reliably discriminate true from false positives and retain clinical usefulness. In low probability patients specificity remains unacceptable and ${ }^{99 m}$ Tc-labelled leucocyte scanning in these patients is of little value as a positive predictor of intestinal inflammation.
\end{abstract}

Scintigraphy of autologous granulocytes using indium-III ("'In) has been well validated as both a specific and sensitive test for the detection of intestinal inflammation. ${ }^{1-4}$ It provides accurate localisation of inflammation, ${ }^{1-5}$ detects complications such as abscess formation, ${ }^{67}$ and, via measurement of faecal "'In excretion, gives a quantitative measure of the degree of inflammation. ${ }^{8}$ Moreover, it has impressive accuracy when employed as a screening test for inflammatory bowel disease (IBD) in patients with abdominal pain and/or diarrhoea. ${ }^{9}$ However, the expense, concerns about radiation microdosimetry, and lack of ready availabilty of "'In in many centres have inhibited its wide adoption and use. Alternative means of radiolabelling of leucocytes have been sought and two apparently satisfactory methods using the readily available $\gamma$-emitting isotope, ${ }^{99 m} \mathrm{Tc}$, have been developed. ${ }^{10-15}$ The images produced by such methods are of generally better resolution than for "'In and the radiation dosage delivered to organs such as the spleen is considerably less. The ability to measure faecal excretion quantitatively, however, is limited ${ }^{1112}$ by the very short half-life of ${ }^{99 \mathrm{~m}} \mathrm{Tc}$.

${ }^{99 m}$ Tc scintigraphy using autologous leucocytes has been evaluated by a number of investigations in patients with a high probability of intestinal inflammation (patients with IBD) and these have indicated that, like "'In-labelled granulocyte scans, they have a high sensitivity of detection of inflammation and perform well in disease localisation. ${ }^{10-14}$ However, no studies have systematically examined their use in disease controls, especially in patients with abdominal symptoms but a low probability of intestinal inflammation, so that specificity may be more accurately assessed. Intestinal accumulation of ${ }^{99 \mathrm{~m}} \mathrm{Tc}$ is reported to occur three or more hours ${ }^{1315-18}$ or even within the first hour ${ }^{19}$ after reinjection of labelled leucocytes in subjects with normal bowels when the hexamethyl propylene amine oxime (HMPAO) method is used but has not been observed when leucocytes are labelled with ${ }^{99 m}$ Tc-stannous colloid. The aims of this study were to examine the ability of positive scans to predict the presence of intestinal inflammation in patients with either a high or low probability of having such inflammation and to compare the performance of the two methods of cell labelling.

\section{Methods}

PATIENT SELECTION

Two groups of patients underwent ${ }^{99 \mathrm{~m}} \mathrm{Tc}$ scintigraphy of autologous leucocytes over a 2.5 year period.

\section{Patients with a high probability of intestinal} inflammation

This group of 29 patients had IBD (26 Crohn's disease, three ulcerative colitis) which had been previously diagnosed on standard clinical, radiological, endoscopic, and pathological criteria. Patients were only included if they had histopathological evidence of disease distribution and activity from biopsies of multiple areas of terminal ileum and colon and/or surgical resection specimens within two weeks of scintigraphy being performed. Assessment of inflammation was made using criteria previously described ${ }^{20}$ and the results recorded as absent, mild, moderate, or severe inflammation. The age of the patients was 17 to 68 (mean 34) years, seven being 
male and 22 female. The disease distribution in patients with Crohn's disease was 12 ileal, six ileocolic, and eight colonic; and in patients with ulcerative colitis, one had pancolitis while the other two had proximal limits of mucosal inflammation at the mid-transverse and upper sigmoid colon respectively.

\section{Patients with a low probability of intestinal inflammation}

This group was further subdivided.

1. Patients with abdominal symptoms possibly related to intestinal inflammation (pain and/or diarrhoea) who were undergoing clinical evaluation ('?IBD' group). This subgroup comprised 37 patients, seven male and 30 female, aged 14 to 69 (mean 33) years. No patient in this group had a definitive diagnosis of IBD. All 17 with positive scans had irritable bowel syndrome diagnosed on a combination of typical history and negative investigations. No patient was taking nonsteroidal anti-inflammatory drugs. Investigations performed included appropriate haematological, biochemical, radiological, endoscopic, and histopathological assessment.

2. Patients with suspected infective focus but without abdominal symptoms referable to the intestine (?'abscess' group). These comprised 21 patients of whom nine were male and 12 female and their ages ranged from 21 to 83 (mean 56) years. Accumulation of ${ }^{99 \mathrm{~m}} \mathrm{Tc}$ in the intestine of these patients was not pursued by specific intestinal investigation. One patient was receiving a non-steroidal anti-inflammatory drug.

\section{SCANNING TECHNIQUES}

\section{Radiopharmaceuticals}

The radiopharmaceuticals used for labelling leucocytes were ${ }^{99 \mathrm{~m}} \mathrm{Tc}$ eluted from an Ansto generator (Sutherland, Australia) and, for some studies, "In oxime, purchased from Amersham

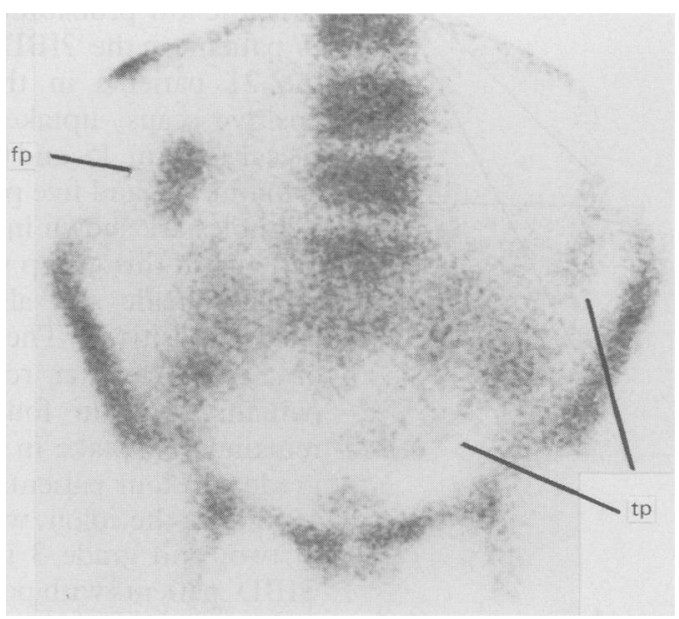

Figure 1: A scan of ${ }^{1 \% m} T$ c-labelled leucocytes at 120 min of a patient with ulcerative colitis. Subtotal colectomy performed one week later revealed severe colitis involving mucosa from the splenic flexure distally, but the remainder of the colon and terminal ileum were normal. Note the true positive ( $t p)$ accumulation of ${ }^{4 \% m} T c$ on the left side of the abdomen correspondng to the inflamed colon which contrasts with the false positive $(f p)$ uptake in the region of the hepatic flexure.

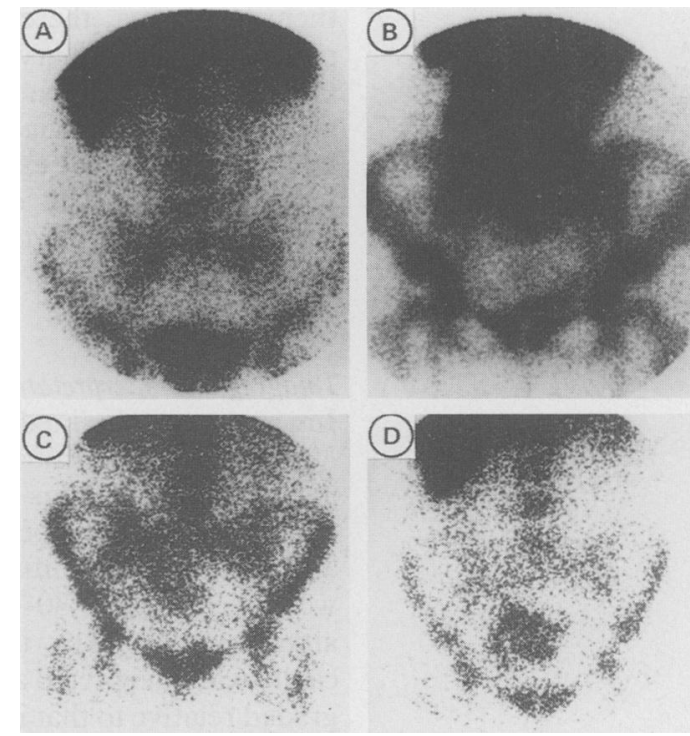

Figure 2: False positive scan of ${ }^{94 m} T c$-labelled leucocytes from two patients. The first patient had a negative scan $(A)$ at 30 min but accumulation of ${ }^{99 m} T c$ was seen in the right side of the colon in the $2 \mathrm{~h}$ image $(B)$. The second patient had uptake in the ileocaecal region at both $45 \min (C)$ and $2 h(D)$.

(Little Chalfont, England). ${ }^{99 m} \mathrm{Tc}$ was complexed to either a stannous fluoride colloid (Radiopharm, Canberra) or to HMPAO as previously described. ${ }^{13}$

\section{Leucocyte labelling}

Leucocytes were labelled with ${ }^{99 m} \mathrm{Tc}$-stannous fluoride colloid as previously reported..$^{1021}$ Briefly, $20 \mathrm{ml}$ of peripheral blood was drawn into a heparinised plastic syringe and incubated for $1 \mathrm{~h}$ with $166-629$ (mean 324 ) $\mathrm{MBq}$ of ${ }^{99 \mathrm{~m}} \mathrm{Tc}$ stannous colloid. Excess colloid was removed by centrifugation and the cells gently resuspended in normal saline and reinfused into the patient. Labelling efficiency was always greater than $95 \%$.

The method for labelling leucocytes with ${ }^{99 m}$ Tc-HMPAO was similar to that previously reported. ${ }^{13}$ Briefly, $50 \mathrm{ml}$ of peripheral blood was collected using acid citrate dextrose (NIH formula A) as anticoagulant and transferred to five $10 \mathrm{ml}$ tubes each containing $0.5 \mathrm{ml}$ of $2 \%$ methylcellulose in normal saline. After sedimentation of red cells for 30-45 min, the supernatant was aspirated and centrifuged at $70-80 \mathrm{~g}$ for $7 \mathrm{~min}$. The cell pellet was resuspended in normal saline and incubated for $15 \mathrm{~min}$ at room temperature with freshly prepared ${ }^{94 \mathrm{~m}} \mathrm{Tc}$ HMPAO. The cells were washed once, resuspended in autologous leucocyte-poor plasma, and returned to the patient. Labelling efficiency (mean (SEM)) was $56(3) \%$ and the patients received 100-592 (mean 285 ) $\mathrm{MBq}$ of ${ }^{9 \% \mathrm{~m}} \mathrm{Tc}$.

"'In labelling of purified granulocytes was carried out by a modification of a previously reported method.". In order to purify granulocytes, leucocytes were first isolated from $50 \mathrm{ml}$ peripheral blood as described above and were then sedimented over a discontinuous FicollPaque (Pharmacia) gradient. The cell pellet was retained, washed twice in autologous plasma, and incubated for 10 mins in $2 \mathrm{ml}$ plasma containing $120 \mathrm{MBq}$ of "'In oxine. After washing 
them in plasma, the cells were returned to the patient. In preliminary experiments, both ${ }^{99 m} \mathrm{Tc}-$ HMPAO-labelled leucocytes and ${ }^{11}$ In-labelled granulocytes had a viability of $>95 \%$, as shown by $0.1 \%$ trypan blue exclusion.

Four patients had dual ${ }^{99 m}$ Tc scans, one by each method. Three patients had "'In scans as well as ${ }^{99 m}$ Tc scans.

\section{Imaging and interpretation}

Images were obtained with a General Electric 300 or 400 or a Toshiba 402A $\gamma$-camera linked to an Informatek Simis III computer. Multiple planar images were performed between $30 \mathrm{~min}$ and $5 \mathrm{~h}$ after cell reinfusion. Commonly, studies were performed at $30-60 \mathrm{~min}, 2-3 \mathrm{~h}$, and 5-6 h after injection. Any uptake in the abdominal cavity was interpreted as a positive study and was graded relative to that in the iliac crests - grade 0 representing no uptake, and 1 less than, 2 equal to, and 3 greater than uptake in the iliac crests. All images were interpreted by one observer (ML) who was blinded to the clinical details.

\section{STATISTICAL EVALUATION}

Incidence data were compared using contingency table analysis with correction for continuity. A p $<0.05$ was considered statistically significant.

\section{Results}

Positive scans were observed in 20 patients with known IBD. Disease distribution was assessed surgically in eight and by histological examination of endoscopic biopsies of the areas of ${ }^{99 \mathrm{~m}} \mathrm{Tc}$ uptake accumulation only in 12 . Uptake of ${ }^{99 \mathrm{~m}} \mathrm{Tc}$ was seen in the ileocaecal region in 13 patients. Three of these were falsely positive (grade 2 in

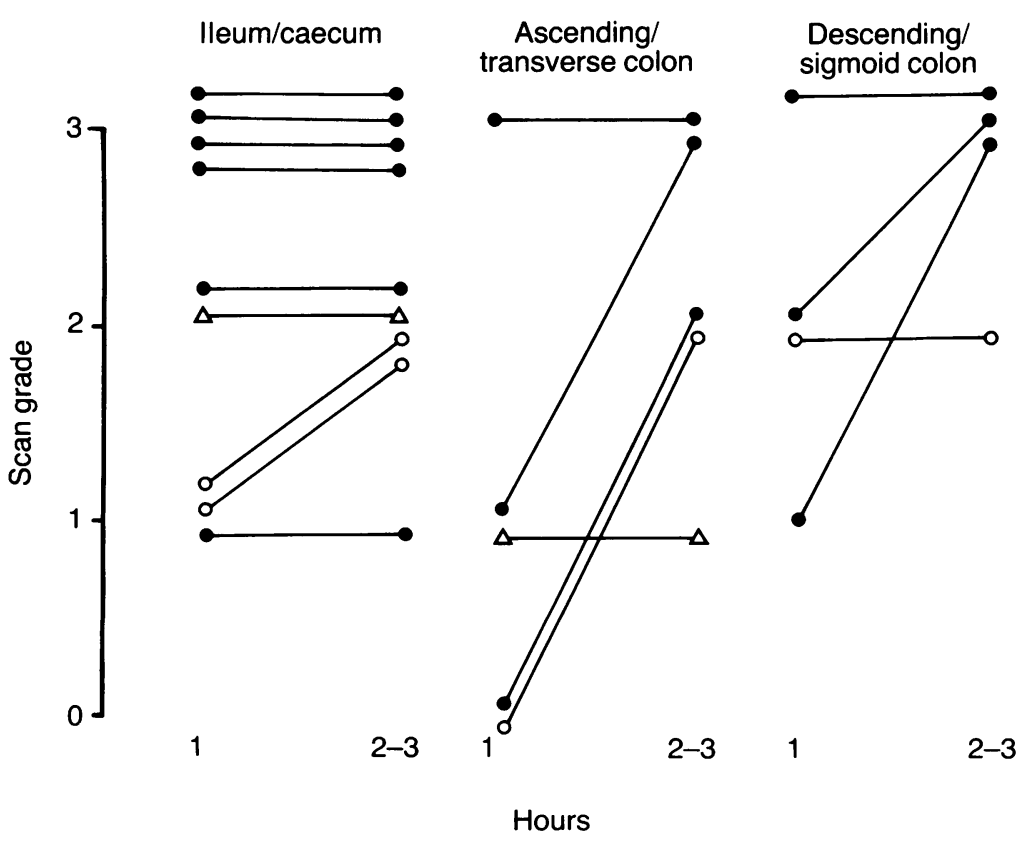

Figure 3: A comparison of ${ }^{4 y_{m}}$ Tc-labelled leucocyte scans performed within the first hour $(1 \mathrm{~h})$ with autologous conventional scans at 2-3 h in terms of grading relative to uptake in the iliac crests and correlation with histological severity. The bowel was subdivided into three region from which biopsies or surgical specimens were histopathologically graded as mildly $(\triangle)$, moderately $(\bigcirc)$, or severely inflamed $(\bigcirc)$. Scan grading was scored as outlined in the text.

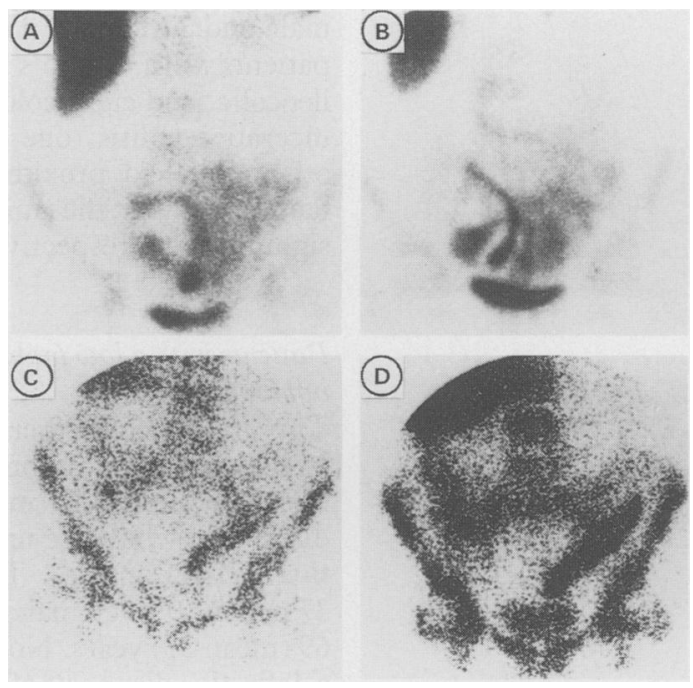

Figure 4: True-positive ${ }^{99 m}$ Tc-labelled leucocyte scans in two patients with Crohn's disease. Scans $(A)$ and $(B)$ were taken at $1 \mathrm{~h}$ and $2 \mathrm{~h}$ respectively in a patient with severe ileitis. Note the similar distribution and grading of ${ }^{99 m} T c$ uptake in both images. Scans $(C)$ and $(D)$ were taken at $1 h$ and $2 h$

respectively in a patient with Crohn's colitis. Note the similar extent of disease but reduced regional grading in the early scan.

two patients and grade 1 in one). In two patients with Crohn's disease, the mucosa of terminal ileum to mid-transverse colon was normal at colonoscopy and ileoscopy, and barium small bowel follow through examination was normal. The third patient had a total colectomy for ulcerative colitis, and the ileum was found to be normal at surgical evaluation and by histology of the ileal stump (Fig 1). Eleven patients had positive scans in regions of the colon other than the caecum but one of these, in a patient who had surgical resection of severely inflamed ileum, was falsely positive (grade 3 ) in the ascending and transverse colon as early as $\mathbf{3 0}$ minutes after reinjection of labelled leucocytes. No patient had false positive ${ }^{99 \mathrm{~m}} \mathrm{Tc}$ accumulation in both anatomical regions. Thus, four (14\%) of the 29 patients with high probability of intestinal inflammation had false positive scans.

In the low probability group, $17(46 \%)$ of the 37 patients in the '?IBD' group and nine (43\%) of the 21 patients in the '?abscess' group had positive scans, uptake being seen in the ileocaecal area in 15 and four patients and in the colon in two and five patients respectively. Two examples are shown in Figure 2. In none of the patients in this group was a definitive diagnosis of IBD made and all scans were apparently falsely positive. The positive images were obtained $4 \mathrm{~h}$ after reinfusion of cells in two patients, $3 \mathrm{~h}$ in four, and $0.5-2 \mathrm{~h}$ in the remainder. Uptake in the ileocaecal region was grade 2 in four patients and grade 3 in the other 15 and, in the colon, was grade 1 in two, grade 2 in two, and grade 3 in three. Investigation of '?IBD' patients with positive scans was thorough and included markers of inflammation (leucocyte count, erythrocyte sedimentation rate and/or C-reactive protein) in all patients, barium studies (10 small bowel, 4 large bowel), flexible sigmoidoscopy (2), colonoscopy (14), and ileoscopy (12). During all endoscopic procedures, multiple biopsies were taken for histopathological assess- 


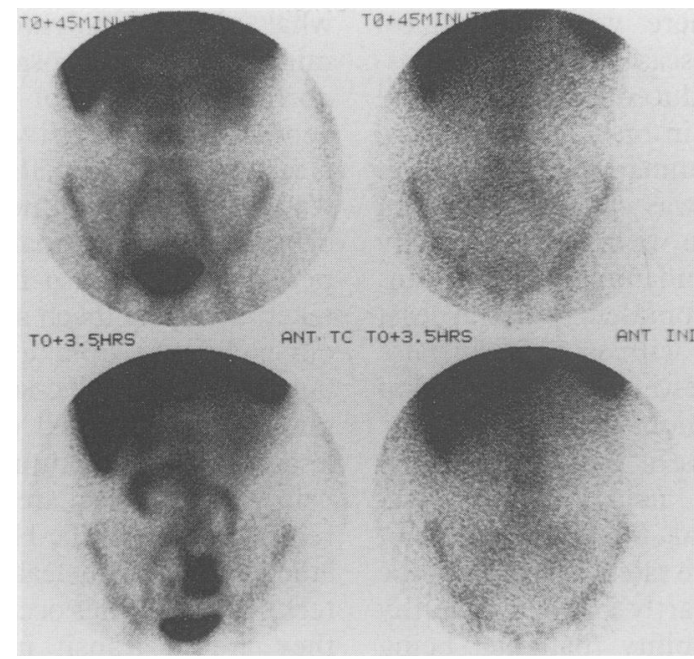

Figure 5: Simultaneous ${ }^{99 m}$ Tc-labelled leucocyte scans (left) and "In-labelled granulocyte scans (right) at 45 min (top) and $3.5 \mathrm{~h}$ (bottom) in a patient with pain in the right iliac fossa secondary to irritable bowel syndrome. Note the accumulation of ${ }^{99 m} T c$ in the small intestine and the simultaneous normal "In image indicating that the ${ }^{99 m} T c$ in the intestinal lumen is unlikely to be cell bound. The $2 h^{99 m} T c$ scan also demonstrated small intestinal uptake while the $22 h^{\prime \prime}$ In scan remained normal (neither shown).

ment. The only abnormal findings comprised one patient with an elevated C-reactive protein and another with a mildly elevated erythrocyte sedimentation rate, both of whom had normal barium follow through studies as well as normal colonoscopy and ileoscopy. In addition, clinical follow up of these patients has exceeded 12 months for 10 without a change from the diagnosis of irritable bowel syndrome. Patients with positive images in the '?abscess' group were not specifically investigated for intestinal inflammation but in none were there clinical indicators of intestinal inflammation. Thus, the false positive rate ( $26 / 58$ patients) in the low probability group was $44 \%$ which was significantly higher than that in the high probability patients $(p=0.011)$.

In an attempt to differentiate false from true positive scans, 27 patients were serially imaged over the first $3 \mathrm{~h}$ to examine the pattern of migration of ${ }^{99 \mathrm{~m}} \mathrm{Tc}$ and the time of first appearance of ${ }^{99 \mathrm{~m}} \mathrm{Tc}$ in the intestine. Neither the rapidity of luminal transit of ${ }^{99 \mathrm{~m}} \mathrm{Tc}$ nor persistence of activity at the original site of uptake were good discriminators. In 15 patients whose scans were false positive, 10 had an initially negative image (within $1 \mathrm{~h}$ of reinjection) and became positive at $2 \mathrm{~h}$ in nine and $3 \mathrm{~h}$ in one. In the other five, the image within the first hour was positive, being grade 1 in one patient and grades 2 and 3 in two patients each. By comparison, in the 12 patients with true positive scans, ${ }^{99 \mathrm{~m}} \mathrm{Tc}$ appeared in the intestine within the first hour in $11(92 \%)$. This was a significantly greater proportion than for false positive scans $(p=0.008)$. Thus, if detection of ${ }^{99 \mathrm{~m}} \mathrm{Tc}$ accumulation only within the first hour was considered positive, the false positive rate would fall to $5 \%$ for high probability and to $15 \%$ for low probability patients while the sensitivity would be reduced by only an estimated $8 \%$.

The accuracy of the true positive images obtained within the first hour was assessed by comparing the regional extent and degree of ${ }^{99 m}$ Tc uptake with those of autologous $2 \mathrm{~h}$ or $3 \mathrm{~h}$ scans and by correlating the scan grade with the histopathological severity in that region. The results are illustrated in Figure 3 and two examples are shown in Figure 4. Scanning within the first hour performed well in the distal small bowel, caecum, and left side of the colon but had reduced sensitivity in the transverse colon where it failed to detect inflammation in two of five patients. The grading of early images with respect to uptake in the iliac crests was, however, worse than $2-3 \mathrm{~h}$ assessment as a predictor of histopathological severity.

The reproducibility of false positive scans was assessed in eight patients who had scans from two days to 20 months apart. In five patients, the original results were reproduced (two days, three days, one month, three months, and 17 months apart) while in three, the image changed, becoming negative in two (three months and 15 months apart) and positive in one ( 20 months apart).

The stannous colloid method for ${ }^{99 \mathrm{~m}} \mathrm{Tc}$-labelling of phagocytic leucocytes was used in 58 patients while the HMPAO method of cell labelling was used in 33 . The false positive rate was similar for each method, being $38 \%$ and $30 \%$ respectively and no differences were seen when high and low probability groups were examined separately. The time of first appearance of ${ }^{99 \mathrm{~m}} \mathrm{Tc}$ in the bowel was also similar, two with the colloid method and three with the HMPAO method becoming positive within the first hour. Four patients had dual scans within four weeks of each other using different labelling methods on each occasion and, in every case, the results were the same. Thus, the false positive problem appeared to be independent of the method used for labelling leucocytes with ${ }^{99 \mathrm{~m}} \mathrm{Tc}$.

As the high specificity and sensitivity of autologous "In-labelled granulocyte scintigraphy to intestinal inflammation has been previously validated, ${ }^{1-4}$ three patients with false positive ${ }^{99 \mathrm{~m}} \mathrm{Tc}$ scans were examined either simultaneously or subsequently within two weeks using "In-labelled cells. On every occasion, the "In scan was negative even in late $(24 \mathrm{~h})$ films (Fig 5)

\section{Discussion}

Of critical importance to the interpretation of the data in this study is the confidence by which intestinal inflammation was excluded in patients with false positive scans. All patients with IBD included in the study had unequivocal assessment of disease distribution and activity and, therefore, false positive scans were investigated and confirmed. In the ?IBD group, mucosal normality was proven in both patients with colonic accumulation (transverse colon and splenic flexure) and 10 of 15 patients with ileocaecal accumulation of ${ }^{99 \mathrm{~m}} \mathrm{Tc}$ by histological examination of biopsies from the suspicious regions of the intestines. Of the remaining five patients, four were radiologically normal whereas one had neither radiological nor endoscopic assessment but had negative scintigraphy of "In-labelled granulocytes. Though specific intestinal investigation was not undertaken in '?abscess' patients 
with positive scans, there were no clinical pointers to intestinal disease except the possibility of ileal disease induced by non-steroidal anti-inflammatory drugs in one. Moreover, the absence of intestinal inflammation in three falsepositive '?IBD' patients was confirmed on "'In scintigraphy, a technique with high sensitivity and specificity for such inflammation. ${ }^{1-4}$ It can, therefore, be said with confidence that at least the majority of patients with apparently false positive scans had no intestinal inflammation at the site of ${ }^{99 \mathrm{~m}} \mathrm{Tc}$ accumulation.

Most published studies of autologous leucocyte ${ }^{99 m}$ Tc scintigraphy using either ${ }^{99 m}$ Tc-

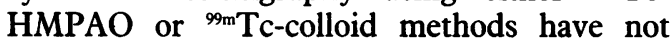
reported high false positive rates. Reasons for the discrepancy may be due partly to too few healthy subjects or low probability patients being examined. In other words, previous reports have dealt almost exclusively with patients carrying a high probability of intestinal inflammation, ${ }^{10-121415}$ many of whom have not had complete histopathological assessment of disease activity in the relevant subregions of the small and large bowel within a short time of imaging. Without these focal examinations, false positive scans may easily be overlooked. In the minority of patients with simultaneous true and false positive sites of ${ }^{99 \mathrm{~m}} \mathrm{Tc}$ accumulation (as demonstrated in Fig 1), it is possible though less likely that rapid luminal transit of ${ }^{99 \mathrm{~m}} \mathrm{Tc}$ either distally or proximally from the inflamed area may explain the incorrect localisation. Normal intestinal accumulation of ${ }^{99 \mathrm{~m}} \mathrm{Tc}$ is known to occur when the HMPAO method is used but only after three or more hours have elapsed after reinjection of the radiolabelled cells ${ }^{1315-18}$ though one study found normal intestinal uptake as early as 30 min. ${ }^{19}$ Such early uptake has not been reported for ${ }^{99 m}$ Tc-colloid labelled leucocytes and has been proposed as a distinct advantage of that labelling method. ${ }^{12}$ Normal intestinal accumulation seen in the present study occurred within $3 \mathrm{~h}$ and, in $15 \%$, within the first hour, figures which closely mimic those of Costa et al for ${ }^{99 \mathrm{~m}} \mathrm{Tc}$ HMPAO labelled cells. ${ }^{19}$ However, this early accumulation occurred independently of the method of tagging ${ }^{99 \mathrm{~m}} \mathrm{Tc}$ to leucocytes.

The possibility that these observations relate to difficulties with technique appears unlikely as the methodology used closely followed the published methods, all cell populations were labelled by a scientist highly experienced in similar celllabelling procedures, and labelling efficiencies were always as high as or higher than published figures. Furthermore, reinjection of dual ("1'Inoxine and ${ }^{99 \mathrm{~m}} \mathrm{Tc}$-colloid) labelled leucocytes in the one patient in whom this procedure was performed demonstrated bowel accumulation of technetium only. In other words, early intestinal accumulation of radiolabel is an inherent problem of ${ }^{99 m} \mathrm{Tc}$-labelled leucocytes and this cannot be overcome by variations of the labelling technique.

How the ${ }^{99 m} \mathrm{Tc}$ reaches the intestinal lumen is uncertain. It is extremely unlikely to be delivered by intact leucocytes as the presence of polymorphonuclear leucocytes, the main subpopulation labelled, in the intestinal mucosa and lumen are features characteristic of inflammation. To what degree polymorphonuclear leucocytes migrate via the mucosa to the intestinal lumen in normal bowel is not known but, in order to generate such intensity of ${ }^{99 \mathrm{~m}} \mathrm{Tc}$ accumulation as is seen in the false positive images, large numbers would be required and these would be plainly obvious to the histopathologist. Free, unbound pertechnitate is also not responsible as this is taken up by stomach and thyroid tissue and, in our experience, uptake in these areas has not been noted during ${ }^{99 \mathrm{~m}} \mathrm{Tc}$-labelled leucocyte scintigraphy (unpublished observations). Nevertheless, soluble technetium species, as evidenced by urinary excretion, are produced in vivo after reinjection of ${ }^{99 \mathrm{~m}} \mathrm{Tc}-\mathrm{HMPAO}$ or ${ }^{99 \mathrm{~m}} \mathrm{Tc}$-colloid labelled cells. If leakage of these unknown technetium species occurs from leucocytes while they are in transit in the bowel, enterocyte uptake and secretion of those species in the lumen is possible. The early luminal appearance of ${ }^{99 m} \mathrm{Tc}$ only in the bowel of the patient who was reinjected with dual labelled leucocytes also suggests elution of a soluble technetium compound in vivo whereas there was no evidence of elution of ${ }^{11} I n$. Suggestions that intestinal accumulation is the result of biliary or gastric secretion seem incorrect as accumulation in the stomach or gall bladder are rare observations in our experience although they appear to be more common in that of others. ${ }^{131617}$ Should such a route be taken, then, on serial imaging, those organs as well as the jejunum should demonstrate uptake at least in a proportion of patients examined. It would seem most likely, therefore, that the ${ }^{99 m} \mathrm{Tc}$ reaches the intestinal lumen via a previously unrecognised transmucosal pathway. Perhaps this pathway is more active in patients with irritable bowel syndrome or severe infections than in normal volunteers who have usually comprised the control population in previous evaluations. ${ }^{10-12}$

With such high false positive rates, the clinical value of ${ }^{99 m} \mathrm{Tc}$-labelled leucocyte scintigraphy in the detection of intestinal inflammation will depend upon the ability to differentiate true from false positive scans. On serial images, the pattern of migration down the intestinal lumen was a poor discriminator. However, ${ }^{99 \mathrm{~m}} \mathrm{Tc}$ accumulation occurs earlier in true positive patients, with significantly more having positive images within the first hour after reinjection of the labelled cells. If interpretation were modified such that only scans with uptake initially seen within the first hour were considered positive, the sensitivity of detection of intestinal inflammation would be reduced by only $8 \%$ while the false positive rate would fall by two-thirds. Thus, in patients with known IBD, the false positive rate would be only $5 \%$ while the high sensitivity, as previously reported ${ }^{10-14}$ and also found by us (unpublished observations), would be only slightly reduced, a performance that is clinically acceptable. Moreover, the assessment of disease extent was similar for the $1 \mathrm{~h}$ scan as for conventional $2 \mathrm{~h}$ and $3 \mathrm{~h}$ scans except for transverse colon. Grading the $1 \mathrm{~h}$ scans relative to iliac crest uptake was, however, inferior to later scans at predicting severity of inflammation. In contrast, a false positive rate of $15 \%$ in low probability patients is still not specific enough 
for ${ }^{99 m} \mathrm{Tc}$-labelled leucocyte scintigraphy to be useful as a predictor of the presence of intestinal inflammation.

In conclusion, a high rate of false positive scans occurs when ${ }^{99 \mathrm{~m}} \mathrm{Tc}$ is used to label autologous leucocytes due to non-specific intestinal accumulation. This rate can be substantially reduced by considering only intestinal uptake within $1 \mathrm{~h}$ of reinjection of labelled cells as truly positive for inflammation. Using this method of interpretation, ${ }^{99 \mathrm{~m}} \mathrm{Tc}$-labelled leucocyte scintigraphy performs well enough to maintain clinical usefulness in patients with known IBD despite a small reduction in sensitivity. However, the scans do not have acceptable specificity in patients with a low probability of intestinal inflammation and, therefore, have no role as positive predictors of inflamed bowel in patients with abdominal symptoms suggestive of IBD.

1 Saverymuttu SH, Peters AM, Hodgson HJF, Chadwick VS, Lavender JP. "Indium autologous leukocyte scanning: comparison with radiology for imaging the colon in inflammatory bowel disease. BMF 1982; 285: 255-7.

2 Saverymuttu SH, Peters AM, Lavender JP, Hodgson HJ, Chadwick VS. "IIIndium autologous leukocytes in inflammatory bowel disease. Gut 1983; 24: 293-9

3 Stein DT, Gray GM, Gregory PB, Anderson M, Goodwin DA, McDougall I. Location and activity of ulcerative and Crohn's colitis by indium-111 leukocyte scan. GastroenterCrohn's colitis by indium

4 Saverymuttu SH, Camilleri M, Rees H, Lavender JP, Hodgson HJF, Chadwick VS. Indium-111 granulocyte scanning in assessment of disease extent and disease activity in inflammatory bowel disease. A comparison with colonoscopy, histology, and fecal indium 111-granulocyte excretion. Gastroenterology 1986; 90: 1121-8.

5 Navab F, Boyd CM, Diner WC, Subramani R, Cahn C. Early and delayed Indium-111 leukocyte imaging in Crohn's disease. Gastroenterology 1987; 93: 829-34.

6 Coleman RE, Black RE, Welch DM, Maxwell JG. Indium-111 labelled leucocytes in the evaluation of suspected abdominal abscesses. Am F Surg 1980; 139: 99-104.

7 Saverymuttu SH, Keshavarzian A, Gibson R, Chadwick VS, Hodgson HJF. Hepatic abscesses associated with Crohn's disease detected by "'indium leukocyte scanning. 7 Clin Gastroenterol 1985; 7: 273-6.

8 Saverymuttu SH, Peters AM, Lavender JP, Pepys MB Hodgson HJF, Chadwick VS. Quantitative fecal indium111 labelled leukocyte excretion in the assessment of diseas activity in Crohn's disease. Gastroenterology 1983; 85: 1333-9.

9 Saverymuttu SH, Peters AM, Crofton ME, et al. 111-Indium granulocytes in the detection of inflammatory bowel disease. Gut 1985; 26: 955-60.

10 Pullman W, Hanna R, Sullivan P, Booth JA, Lomas F, Doe WF. Technetium-99m autologous phagocyte scanning: a new imaging technique for inflammatory bowel disease. $B M 7$ 1986; 293: 171-4.

11 Bartholomeusz DL, Shearman DJC, Chatterton BE, et al. Assessment of disease activity and localization in inflammatory bowel disease using ${ }^{{ }^{4} m_{m}}$ Tc-labelled leucocytes. $\mathcal{F}$

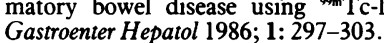

12 Pullman WE, Sullivan PJ, Barratt PJ, Lising J, Booth JA, Doe WF. Assessment of inflammatory bowel disease activity by technetium-99m phagocyte scanning. Gastroenterology 1988 ; 95: 989-96

13 Roddie ME, Peters AM, Danpure HJ, et al. Inflammation: imaging with Tc-99m HMPAO-labelled leukocytes. Radialogy 1988; 166: 767-72.

14 Scholmerich J, Schmidt E, Schumichen C, Billmann P Schmidt. H, Gerok W. Scintigraphic assessment of bowel involvement and disease activity in Crohn's disease using involvement and disease activity in Crohn's disease using technetium 99m-hexamethyl propylene amine oxin
leukocyte label. Gastroenterology 1988; 95: 1287-93.

15 Peters AM. Imaging with white cells. Clin Radiol 1989; 40: 453-4.

16 Becker W, Schomann E, Fischbach W, Borner W, Gruner KR. Comparison of ${ }^{\circ} \mathrm{Tc}^{\mathrm{m}}-\mathrm{HMPAO}$ and "In-oxime labelled granulocytes in man: first clinical results. Nucl Med Commun 1988; 9: 435-7.

17 Peters AM, Roddie ME, Danpure HJ, et al. ${ }^{\text {*T }} \mathrm{Tc}^{\mathrm{m}}-\mathrm{HMPAO}$ labelled leucocytes: comparison with "'In-tropolonate labelled granulocytes. Nucl Med Commun 1988; 9: 449-63.

18 Mountford PJ, Kettle AG, O'Doherty MJ, Coakley AJ Comparison of technetium-99m-HMPAO leukocytes with indium-111-oxine leukocytes for localizing intraabdominal indium-111-oxine leukocytes for local
sepsis. 7 Nucl Med 1990; 31: 311-5.

19 Costa DC, Lui D, Ell PJ. White cells radiolabelled with "'In and ${ }^{*} \mathrm{~T}^{\mathrm{m}}$ - a study of relative sensitivity and in vivo viability. Nucl Med Commun 1988; 9: 725-31.

20 Gibson PR, Van de Pol E, Barratt PJ, Doe WF. Ulcerative colitis - a disease characterised by the abnormal colonic epithelial cell? Gut 1988; 29: 516-21.

21 Hanna R, Braun T, Levendel A, Lomas F. Radiochemistry and biostability of autologous leucocytes labelled with ${ }^{4 \% m} \mathrm{~T} C-$ stannous colloid in whole blood. Eur $\mathcal{F}$ Nucl Med 1984; 9 . 216-9.

22 Thakur ML, Lavender JP, Arnot RN, Silvester DJ, Segal AW. Indium"'-labelled autologous leucocytes in man. f Nucl Med 1977; 18: 1014-21. 